\title{
Intramural Metastatic Squamous Cell Carcinoma of Stomach
}

\author{
Arulprakash Sarangapani • Rathnakar Kini • \\ S. Chitra $\cdot$ T. Pugazhendhi $\cdot$ S. Jeevan Kumar
}

Received: 25 November 2008 / Accepted: 17 March 2009/Published online: 19 April 2011

(C) Association of Surgeons of India 2011

\begin{abstract}
Intramural metastasis to the stomach (IMMS) from carcinoma esophagus, excluding direct invasion and spread to the stomach, is relatively uncommon. Diagnosis of IMMS is based on histological criteria, and it is associated with poor prognosis (Takubo et al. Cancer 65:1816-1819, 1990]. We report two patients of SCC oesophagus in whom intramural metastasis to stomach has occurred.
\end{abstract}

Keywords Squamous cell carcinoma stomach - Metastasis stomach $\cdot$ Intramural metastasis stomach

Case 1: 60 year old female presented with complains of pain abdomen, non-bilious vomiting of two weeks duration. She was treated for well differentiated squamous cell carcinoma esophagus (lower third) 7 months back. She underwent tanshiatal esophagectomy, gastric pull through and esophago-gastric anastomosis. Patient remained asymptomatic since then. Ultrasound abdomen and CT scan thorax and abdomen did not reveal any metastasis at initial presentation. Endoscopic examination showed an excavating ulcer (Fig. 1) along the lesser curve of the stomach, biopsy was taken. Histopathological examination showed malignant squamous epithelial cells, keratin pearl formation, areas of hemorrhage surrounded by gastric cells and was suggestive of well differentiated squamous cell carcinoma (Fig. 2). Patient was given chemotherapy (patient refused surgery), but succumbed after 2 months of treatment.

Case 2: 35 year old man presented with complains of pain abdomen, non-bilious vomiting, hematemesis, poor

Arulprakash S. $(\bowtie) \cdot$ Rathnakar Kini $\cdot$ S. Chitra $\cdot$ T. Pugazhendhi $\cdot$

S. Jeevan Kumar

Department of Digestive Health and Diseases,

Kilpauk Medical College,

Chennai, India

e-mail: drarulaash@yahoo.co.in appetite and loss of weight. He received radiotherapy for well differentiated squamous cell carcinoma esophagus upper third 6 months before. CT scan abdomen revealed extensive liver secondaries. Endoscopic examination showed a large excavating ulcer (Fig. 3) in fundus of the stomach, biopsy was taken. Histopathological examination was suggestive of well differentiated squamous cell carcinoma. He was started on chemotherapy but succumbed after 1 month of treatment.

\section{Discussion}

The most critical reasons for poor prognosis in SCC esophagus are that metastatic spread is quite common. The most frequent site of visceral metastases is to the liver, followed by lung and adrenals. The presence of metastases within the stomach from esophageal carcinoma is rare. The incidence ranges from $0 \%$ to $15 \%$ in autopsy cases [2]. Intramural metastasis (IMM) is defined as a metastatic tumor in the oesophageal or gastric wall arising from a primary lesion in the oesophagus, and upon histological examination, the secondary tumor mass is found to be (a) clearly separate from the primary tumor, (b) located in the oesophageal or gastric wall, (c) not surrounded by endothelium, and (d) not accompanied by an intraepithelial cancerous extension. These features distinguish IMM from a tumor embolus in a vessel, from multiple primary lesions in the oesophagus, and from a protrusion of the invasive primary tumor [3]. Our diagnosis was based on these criteria.

Stomach metastasis is induced mainly via the lymphatic route rather than via the bloodstream route that is common in other types of distant organ metastasis. IMM of oesophageal cancer was first reported by Watson [4] in 1933, and has been considered to be an important mechanism of tumor spread - extension by way of the 


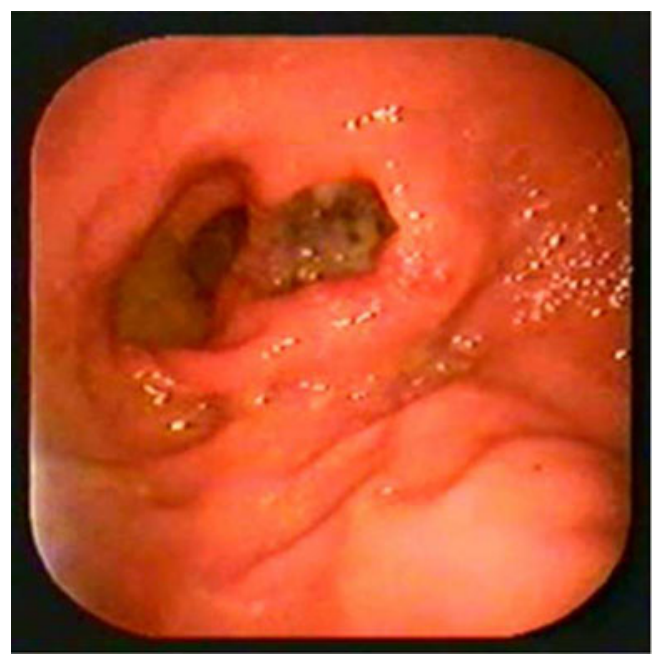

Fig. 1 CASE 1: Endoscopic view: An excavating ulcer along the lesser curve of the stomach

submucous lymphatics. Weinberg [5] documented an instance of lymphatic communication between the stomach and oesophagus by the growth of new lymphatic channels into the carcinomatous lesion. IMM Stomach does not correlate with histological type but lymphatic invasion. Almost all reported cases had metastatic tumors located in the upper onethird of the stomach, which was true in our cases.

Esophageal carcinoma with distant organ metastases at the time of the operation seems to be a systemic disease even if the primary lesion is resectable. Stomach metastasis (via lymphatic permeation) differs somewhat from metastasis to other distant organs (hematogenous route). Stomach metastases had a better prognosis than other distant organ metastases [2]. Although the depth of an oesophageal cancer may be T1, the possibility of lymphatic invasion still exists, and thus the existence of lymph metastasis and IMM

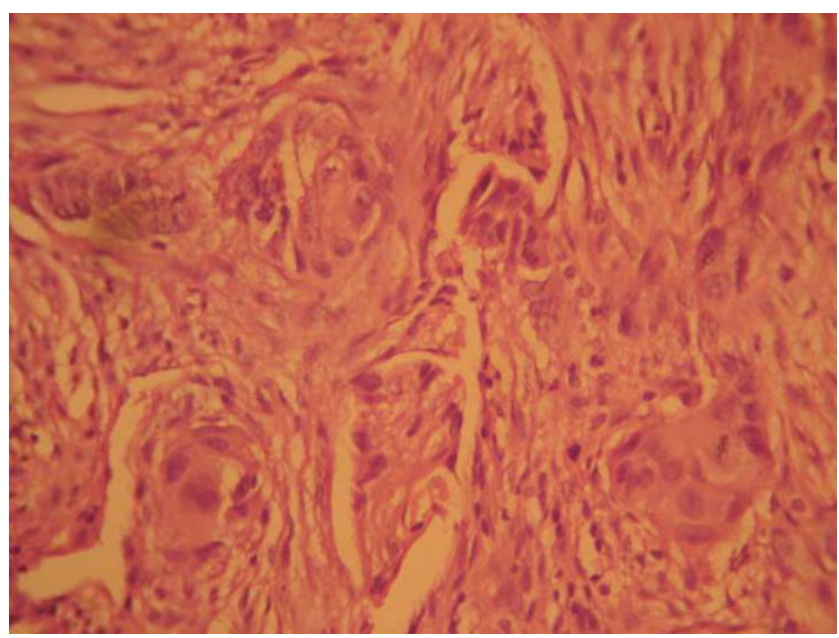

Fig. 2 Histopathology slide: Malignant squamous epithelial cells, keratin pearls, areas of hemorrhage surrounded by gastric cells suggestive of well differentiated squamous cell carcinoma

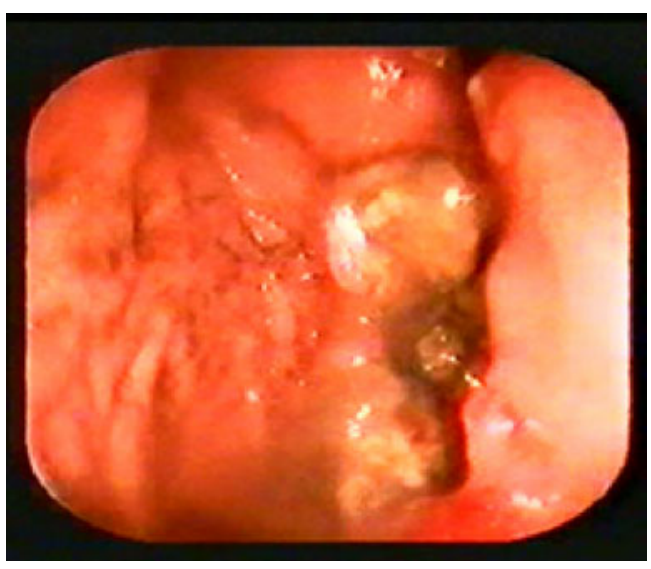

Fig. 3 Case 2: Endoscopic view: A large excavating ulcer in fundus of on retroflexion

including to the stomach must be carefully determined. Treatment modality includes surgical resection, radiation therapy, chemotherapy, or some combination of these. Both of our patients where given chemotherapy, but died within 2 months. When the metastatic tumor is limited to the stomach and is small $<2 \mathrm{~cm}$ in diameter, surgical resection should be performed. However, if the metastatic tumor is larger than $2 \mathrm{~cm}$, surgery is no doubt an unsatisfactory palliative therapy in most patients [2]. Presence of intramural metastasis is an important factor to consider when evaluating the prognosis of patients with SCC of the esophagus [6].

\section{Conclusion}

Intramural metastatic squamous cell carcinoma of stomach from carcinoma esophagus is an uncommon entity and important in prognosis. It is most important that, in patients with esophageal carcinoma, the stomach is examined accurately and carefully to diagnose small metastases.

\section{References}

1. Takubo K, Sasajima K, Yamashita K, Tanaka Y, Fujita K (1990) Prognostic significance of intramural metastasis in patients with esophageal carcinoma. Cancer 65:1816-1819

2. Saito T, lizuka T, Kato H, Kato H, Watanabe H (1985) Esophageal carcinoma metastatic to the stomach: a clinicopathological study of 35 cases. Cancer 56:2235-2241

3. Kato $\mathrm{H}$, Tachimori $\mathrm{Y}$, Watanabe $\mathrm{H}$, Itabashi $\mathrm{M}$, Hirota $\mathrm{T}$, Yamaguchi H, Ishikawa T (1992) Intramural metastasis of thoracic esophageal carcinoma. Int J Cancer 50:49-52

4. Watson WL (1933) Carcinoma of the esophagus. Surg Gynecol Obstet 56:884-897

5. Weinberg JA (1972) The lymphatics in cancer. Saunders, Philadelphia, pp 245-247

6. Kato H, Tachimori Y, Watanabe H, Itabashi M, Hirota T, Yamaguchi H, Ishikawa T (1992) Intramural metastasis of thoracic esophageal carcinoma. Int J Cancer 50(1):49-52 